\title{
Getting embryonic stem cell therapy right
}

\author{
Embryonic stem cell therapy may soon enter clinical trials. The US Food and Drug Administration (FDA) will \\ need to provide clear guidance on how it will regulate such trials and ensure that politics will not cloud the \\ regulatory process.
}

S ince the derivation of human embryonic stem cells in the 1990s, they have been touted for their potential to cure a wide range of diseases. Now we may soon get the chance to see what these cells can do. With several biotechnology companies getting ready to move embryonic stem cell therapy into the clinic, the FDA convened a public advisory committee meeting on 10 April to gather information about safety concerns for this type of therapy. In the meeting, three companies outlined their most advanced programs for using embryonic stem cell therapy-Geron for the treatment of acute spinal cord injury, Novocell for diabetes and Advanced Cell Technology for macular degeneration.

In view of the intrinsic ability of embryonic stem cells to form teratomas - a type of tumor-the major safety issue raised at the FDA's meeting was how to ensure the nontumorigenicity of the cells used for therapy. For the proposed therapies, embryonic stem cells are first forced in tissue culture to differentiate into an appropriate cell type for the particular disease being treated-for example, retinal pigment epithelial cells for treating macular degeneration. It is these more differentiated cells that are injected into patients. The purity of the differentiated cells is thus a crucial safety concern. Embryonic stem cells are known to have a high propensity to adapt to tissue culture conditions-for instance, by becoming aneuploid-which only increases the need for thorough characterization of the cells used for treatment.

For some diseases, it may be desirable to differentiate the embryonic stem cells into a progenitor cell type with intermediate developmental plasticity between embryonic stem cells and terminally differentiated cells. In this case, particular care will be needed to assure that the progenitor cells will not form tumors.

As discussed at the FDA's meeting, selection of the most appropriate preclinical models for demonstrating the nontumorigenicity of the injected cells will be a key issue. Because treatment of different diseases involves different cell types, with different routes of administration and delivery into different locations, the preclinical models used will need to be disease- and cell type-specific. Another important issue is how best to monitor patients for tumor formation, which would presumably need to be done for the rest of their lives.

As with other types of cell therapy in which the cells used are not derived from the patient being treated, the question of the potential immunogenicity of the cells will also need to be addressed.

With the glare of the spotlight on anything having to do with embryonic stem cells, a high degree of caution seems appropriate in early clinical trials. As amply demonstrated by the trials and tribulations of gene therapy, it serves no one's interest to move into the clinic before safety concerns are thoroughly considered. At the same time, there is risk in any new type of treatment, and the FDA does have considerable experience with other types of cell therapy, such as hematopoietic stem cell transplantation.

The FDA's meeting comes at a time when recent news on stem cells has been dominated by discoveries using induced pluripotent cells-fibroblasts reprogrammed by introduced genes to have many of the properties of embryonic stem cells. In principle, induced pluripotent cells can be derived from the patient being treated, obviating immunogenicity concerns. These cells also seem free of the ethical and political issues that beset work on embryonic stem cells. Although the popular media may have given the impression that, with the advent of induced pluripotent cells, embryonic stem cells are on the verge of obsolescence, this meeting indicates quite the opposite.

In view of the antagonism of the current US administration to research using embryonic stem cells, we are encouraged by the willingness of the FDA to have an open discussion on how these cells can be moved into the clinic. It's not yet clear when the first clinical trials for embryonic stem cell therapy might start. When they do, it's certain that the ethical and political debate on the use of these cells will heat up, and the FDA will need to maintain a politically neutral stance. A demonstrated benefit of embryonic stem cell therapy in patients would go a long way toward settling this debate. 